\title{
TEORÍA, INVESTIGACIÓN Y APLICACIONES CLÍNICAS DEL AUTOCONTROL
}

\author{
William Montgomery URDaY ${ }^{1}$ \\ Universidad Nacional Mayor de San Marcos, Lima, Perú \\ (RECIBIDO EL 31/10/2008, ACEPTADO EL $9 / 12 / 2008$ )
}

\begin{abstract}
RESUMEN
Se examina el papel del autocontrol en su variedad de significados y aplicaciones, desde la experimentación hasta la clínica conductual. Se enfatiza que los repertorios conductuales que reflejan el fenómeno de autocontrol en un individuo son aprendidos, lo que fortalece la necesidad de promover su aprendizaje en la situación psicoterapéutica mediante procedimientos estructurados para ello, tales como las terapias de manejo de la ansiedad y la ira, de asertividad, de condicionamiento encubierto, de reestructuración racional, de solución de problemas, de entrenamiento autoinstruccional y de biorretroalimentación, propiciando a través del desarrollo de esos procedimientos la autovigilancia y la relajación, las destrezas de afrontamiento, y cambios específicos en el estilo de vida.
\end{abstract}

Palabras clave: Autocontrol, terapia conductual, personalidad, afrontamiento, control.

\begin{abstract}
It examines the role of self-control in its variety of meanings and applications, from experimentation to clinical behavior. It is emphasized that behavioral repertoires of selfcontrol in an individual are learned, which reinforces the need to promote learning in psychotherapeutic situation through structured procedures for doing so, such as therapies for management of anxiety and anger, assertiveness, covert conditioning, rational restructuring, problem solving, autoinstruccional training, and biofeedback, propitiating through development of such procedures the self-monitoring and relaxation, coping skills, and specific changes in lifestyle.
\end{abstract}

Keywords: Self-control, behavioral therapy, personality, coping, control.

1 Docente Asociado de la Facultad de Psicología de la UNMSM. E-mail: avidolector@yahoo.es 
Cualquier concepción que sostuviera que no tomamos parte en la determinación de lo que hacemos, falla desde el principio.

Arthur. W. Staats (1975, 1979: 421)

Cuestión de larga data en el análisis del comportamiento humaño es el problema vinculado al ejercicio de la autonomía personal dentro de un marco de tipo determinista. Este problema es relevante no sólo por razones filosóficas, sino también por sus correlatos aplicados. Especialmente en referencia al uso efectivo de procedimientos de autorregulación y autocontrol por parte del cliente (autónomo) bajo consulta en el episodio psicoterapéutico (Montgomery, 1997).

Como dice Rehm (1991), la noción de autocontrol no es una teoría sistemática única de la conducta humana, por eso tiene correlatos de varios tipos. Uno de ellos es filosófico y teológico, porque converge con el ejercicio del libre albedrío sobre las propias acciones. $\mathrm{Al}$ respecto, Kanfer y Phillips $(1970,1980)$ señalan que el primer relato de uso del autocontrol es el hecho por Homero sobre Ulises quien se coloca cera en los oídos para no escuchar a las sirenas. Por otra parte se encuentran también claras referencias a la autodirección personal en varios textos religiosos (la epístola del apóstol San Pablo a los Gálatas, la de San Pedro a Tito, el capítulo III del libro VII en las Confesiones de San Agustín, etc.).

El propio Skinner relata cómo es que se le ocurrió la idea del autocontrol al escuchar la emisión radial de un predicador evangélico que establecía una distinción entre personalidades controladas y controladoras. Al responder a cierta persona que lamentaba no poder dominar su afición a la bebida, el predicador le dijo: “¿Qué quiere decir esto de que no sabe cortar su afición a la bebida? ¿No es acaso su brazo el que levante el vaso hasta sus labios? ¿Quiere decir que no puede dominar su brazo?” (Skinner, 1979, 1980: 105). También en la literatura no cristiana se advierten ideas semejantes, como por ejemplo en la religión hindú, donde los elementos esenciales son dos: el conocimiento de sí mismo y el autocontrol de las apetencias.

Estando la noción de autocontrol, y, por consiguiente, de libre albedrío relacionada estrechamente con la ética y la vida cotidiana, es natural que también se muestre como relevante en lo sociopolítico, socioeconómico y psicológico, al subrayarse su importancia respecto a la contraposición entre sistemas democráticos o autoritarios, economía liberal o dirigida, y orgañocentrismo adversus ambientalismo. Por ejemplo, los resultados experimentales de una teoría psicológica, la de la reactancia (Brehm, cit. por Peña, 2004: 72), sugieren que el despojo ilusorio o real de alguna libertad de respuesta motiva en el individuo un estado dirigido a su restauración.

Lo cierto es que la controversia histórica entre quienes entienden filosóficamente la libertad como voluntad de proceder sin cortapisas, y quienes la entienden como una ilusión, resulta improductiva. Para efectos de este escrito semejante contraposición es simplista, inadecuada e inútil. Los repertorios que se consideran tradicionalmente dentro de los fueros del "libre albedrío" son aprendidos. Si bien se trata de un valor relativo predeterminado por circunstancias exteriores, la libertad es real para quien la ejerce como comportamiento moldeado para analizar, decidir, y modificar las condiciones que lo rodean. En suma, 
"la libertad, la autodirección y la espontaneidad son... lo que experimentamos en nuestra propia conducta. Nuestros repertorios somos nosotros. Son nuestras individualidades, nuestro «ser»" (Staats, 1975, 1979: 429). La causación personal es, pues, tan posible como reproducible. Los individuos aprenden a ejercer su voluntad y por lo tanto a autodirigirse con diversos grados de eficiencia y efectividad, y si se necesita se pueden incrementar esos niveles.

El objetivo de este artículo es discutir dicha cuestión desde una óptica suficientemente amplia como para abarcar la variedad de significados y aplicaciones del término "autocontrol", desde la experimentación hasta la clínica conductual.

\section{LAS CONNOTACIONES DEL CONTROL}

\section{El control experimental}

Como es conocido, al interior del análisis conductual de orientación skinneriana el término "control" se ha identificado frecuentemente con operaciones relativas al manejo preciso de una variable que probabiliza cambios en las tasas de respuestas de organismos pertenecientes a diversas cadenas evolutivas, dentro de una situación experimental. Desde esta óptica, "El control podría entenderse como una forma de incrementar la capacidad de predicción de un organismo (sobre su entorno) mediante su actividad" (Peña, 2004: 71).

(Es así que se utiliza ese tipo de control tanto con especies distintas a la humana (véase, por ejemplo, Ardoy, 2007) como en la investigación acerca de actividades correctivas con seres humaños, especialmente dentro del ámbito de la conducta lectiva, tales como el reforzamiento de la imitación, la lectura, la disciplina, el aseo y el tratamiento de defectos lingüísticos. Esto es reseñado por Kevin (2008), con motivo de los cincuenta años de la revista Journal of Experimental Analisis of Behavior. En esa publicación tan característica también se puede ver que, inclusive en la actual experimentación con individuos humaños, se indaga sobre operaciones básicas de reforzamiento y castigo para calibrar su influencia en la toma de decisiones (Rasmussen y Newland, 2008), lo que da una idea de lo arraigada que está semejante forma de búsqueda de control.

El caudal de datos a nivel experimental es vasto, utilizándose varias maneras de "controlar" diseños contingenciales. Por ejemplo, mediante cámaras experimentales acopladas donde la conducta de uno de dos sujetos se usa para programar contingencias que se presentan al otro. O también mediante la manipulación de variadas formas de control de estímulo (exteroceptivo, interoceptivo, inhibitorio, etc.) como señales discriminativas.

\section{El control social}

Por otro lado, también hay controles no necesariamente planificados. Eso sucede con respecto a las contingencias naturales azarosas y al control social. En general, este último tipo de control se verifica a través de una relación en la cual una persona o grupo de personas modifica directa o indirectamente la conducta de otra, lo que puede suceder por medio del manejo estimular reforzante o aversivo, del uso del castigo, del señalamiento 
de contingencias, de la provocación de estados emocionales o estados de privación o de saciedad, y a través de la inoculación de sustancias químicas. Desde este punto de vista, es imposible no estar sujeto a algún tipo de control en la vida cotidiana (Skinner, 1953, 1971).

\section{El control psicoterapéutico}

Lo dicho en el anterior parágrafo es más válido aún en la consulta psicoterapéutica. La aplicación del control en la clínica por parte de la acción discriminativa, evocadora y reforzante del prestatario del servicio se lleva a cabo mediante procedimientos tales como: a) la instigación oral o física, textual, gráfica o gestual, b) la retroalimentación de las ejecuciones deseables, c) el moldeamiento de performances adecuadas, y d) la prescripción instruccional (Montgomery, 2002).

A través de tales métodos, se procura perfeccionar los desempeños del usuario del servicio con respecto a los objetivos del tratamiento, y en especial la auto-observación y automedición de sus funciones fisiológicas y somáticas, la fuerza de sus repertorios, y por fin inducir el análisis funcional de su conducta en relación con su circunstancia problema. Esto conlleva, como lo indica el estudio clásico de Ferster en 1965 (cit. por Campos, 1973: 56), el fomento terapéutico de una forma de control mediante la cual la misma persona bajo intervención pueda, en ausencia del terapeuta, alterar su propia relación inadecuada con el medio, incrementar su efectividad de interacción productiva futura, y cambiar su entorno físico y social. En otras palabras, el ejercicio del autocontrol y la consiguiente autodirección.

\section{EL AUTOCONTROL}

Thoresen y Mahoney $(1974,1980)$ definen funcionalmente el autocontrol como el fenómeno en que aquella conducta que ha tenido una probabilidad previa menor de ocurrencia que la de otras alternativas disponibles, es de repente emitida en ausencia relativa de impedimentos externos inmediatos. Por otra parte, en uno de los capítulos de Ciencia y conducta humana, Skinner (1953/1971) categoriza algunas clases de autocontrol:

1) Restricción y ayuda física, por ejemplo cuando alguien se lleva la maño a la boca para sofocar la risa, o también cuando alguien utiliza un micrófono para ampliar la potencia de su voz ante un auditorio.

2) Manipulación de estados de privación y saciedad, propiciando condiciones previas para incentivar o extinguir comportamientos de búsqueda de refuerzo. Por ejemplo, al no comer nada las horas anteriores a un banquete con el objeto de tener más hambre al llegar a él.

3) Manipulación de estados emocionales, autoinduciendo cambios en ellos, por ejemplo cuando se cuanta hasta diez antes de responder de manera airada.

4) Uso de estímulos aversivos, como el reloj despertador del cual sólo de puede "escapar" levantándose. 
5) Uso de fármacos o estimulantes (por ejemplo un analgésico, o la cafeína).

6) Autorreforzamiento o autoextinción, como cuando alguien se reúne o (en el caso inverso evita) reunirse continuamente con amigos que sabe que van a elogiar su conducta.

7) Autocastigo de las respuestas indeseables, como es el caso de la autoflagelación que algunos monjes se administran para sofocar los deseos de "pecar".

8) Contrarrestando la conducta no deseada con respuestas incompatibles. Ejemplificando sobre ello, un pasaje de Los miserables describe una costumbre de las monjas en los conventos, según la cual a cada hora del día la campana da una señal para que todas interrumpan lo que dicen, hacen o piensan, y dicen al unísono: "A todas horas bendito y alabado sea el Santísimo...", etc., con el fin de desviar su atención de la vida mundana.

\section{Connotaciones del autocontrol}

Más allá de las disquisiciones sobre el ejercicio del autocontrol como una conducta que describe, analiza y opera sobre otra conducta del mismo individuo, hay quienes procuran aclarar su connotación como lo indica Rezk (1976), cuya reseña de algunos de los aportes en ese sentido seguiremos.

Los términos de automanejo y autorregulación son casos alternos. El automanejo involucra (según lo definen Cautela y también Mahoney) una respuesta que modifica otra en cuanto a sus tasas de frecuencia. La autorregulación, por su parte, como la usa Kanfer, requiere que se muestre un patrón comportamental en ausencia relativa de estimulación inmediata, en tres fases: automanejo, autoevaluación y autorefuerzo.

A su vez, el autocontrol, propiamente dicho, puede entenderse de dos formas: como cambio autoimpuesto (cautela) y como autorrestricción o control de la propia impulsividad (Kanfer). Ahora bien, esto último no puede ser absolutizado ya que a veces la impulsividad es la respuesta más adaptativa, y un individuo común debería tener la capacidad de ser impulsivo o controlado en función de qué es lo más adaptable de acuerdo a cada situación (Logue, 1995).

\section{El autocontrol experimental}

Como en el caso del control, se han hecho múltiples estudios experimentales para investigar el autocontrol animal, comparándolo con el que sujetos humaños pueden ejercer, sobre todo en cuanto al autocontrol de la impulsividad. Por ejemplo Jackson y Hackenberg (1996) utilizaron palomas, y Green y Estle (2003) ratas. Los resultados reportan respuestas muy parecidas en las tres especies. El planteamiento típico es el que tiene que ver con el "paradigma de tentación": poner a disposición del sujeto la administración de reforzadores de acuerdo a reglas de refuerzo inmediato (o contingente) adversus refuerzo demorado, que regulan los estándares de su ejecutoria. Dos respuestas de elección pueden ser emitidas, una inmediata lleva a un menor refuerzo con un pequeño retraso, otra de espera lleva a un 
mayor refuerzo con un retraso mayor. La tendencia general de respuesta es concordante en particular con la famosa investigación liderada por Walter Mischel en los años 60 (ver la descripción detallada en Grosch y Neuringer, 1981; Ameriks, Caplin, Leahy \& Tyler, 2004), en la cual propuso a un grupo de niños de cuatro años que podían tomar un malvavisco inmediatamente o esperar unos minutos y coger dos. Los más "impulsivos" cogían rápidamente el dulce ofrecido.

Mischel y sus colegas siguieron observando durante catorce años la conducta de esos niños, hallando que los "impulsivos" tenían baja autoestima y poca tolerancia a la frustración, mientras que los que habían esperado tendían a mostrar más competencia social y éxito académico, lo que denota la importancia clínica de este hallazgo.

También es posible examinar las diferencias entre los individuos en el desarrollo del autocontrol mediante el examen de la función de la cultura de referencia. Puesto que el aprendizaje social es particular a cada cultura y a cada etnia, es posible que durante el desarrollo las personas que crecen en los diferentes medio ambientes humaños adquieran diferentes grados o tipos de autocontrol, como se infiere de la comparación gruesa entre los rasgos culturales de los hemisferios occidental y oriental del mundo, e incluso entre parcelas de una misma región.

En las sociedades orientales se privilegia en general una forma de autocontrol colectivo muy distinto a lo predominante en los Estados americaños, que es el individualismo. La mayoría de japoneses y chinos, por ejemplo, dejan de lado sus intereses personales a favor de objetivos sociales comunes, considerando que la gratificación individual sólo es aceptable si todos participan del progreso (Avia, 1995; Logue, 1995). Otra modalidad tradicional de autocontrol es, como en la India, identificada con la aceptación de las circunstancias que tocan vivir a cada cual, y el doblegamiento ante condiciones que no se pueden cambiar. Por cierto que esto no constituye una modalidad de "indefensión aprendida". Al contrario, permite a los individuos adaptarse mejor a situaciones difíciles, e incluso sacar buen partido de ellas (el bienestar emocional).

\section{AUTOCONTROL, PERSONALIDAD Y MOTIVACIÓN}

El ejercicio del autocontrol se suele vincular a un agente controlador que se llama "sí mismo" o "yo" en la literatura psicológica, que resulta ser una abstracción representativa del conjunto de repertorios funcionalmente unificado que integra el individuo (Skinner, 1953/1971). Dichos repertorios son aprendidos y están unidos en base a una historia de reforzamiento común, o bien se organizan en torno a situaciones particulares, siendo combinados e interrelacionados gracias al lenguaje (Kanfer y Phillips, 1970, 1980). Desde este punto de vista, la personalidad viene a ser algo similar.

Conceptos desarrollados al amparo de esa relación entre el autocontrol y la personalidad son los constructos de locus de control y autoeficacia percibida.

A su vez, el constructo de necesidad de control se vincula con el área de la motivación. 


\section{El locus de control}

En la concepción de J. B. Rotter es central la expectativa como percepción predictora del comportamiento. De esta manera, dio por sentado que si el individuo percibe una correspondencia relativamente permanente entre su conducta y los cambios contingentes de una situación, atribuye los resultados a su ejercicio de control interno. Si, por el contrario, percibe que tal correspondencia es sólo ocasional o parcial, atribuye los resultados a la suerte. Los correlatos de esta construcción explicativa para la personalidad son evidentes: un locus de control interno implica mayor responsabilidad y sentimiento de que uno puede influir en las circunstancias, con todas las implicancias autorregulatorias que eso tiene a nivel conductual (Visdómine y Luciaño, 2006).

\section{La autoeficacia percibida}

Según Bandura $(1986,1987)$, la manera cómo las personas interpretan los cambios producidos por sus acciones les informa sobre las consecuencias ambientales, y permite alterar tanto su entorno como sus propias creencias. Estas creencias que tienen los individuos sobre sí mismos son de singular importancia para el ejercicio del control y el desarrollo de su competencia personal, permitiéndoles manejarse con eficacia. En suma, el conjunto de juicios que la gente hace respecto a su propia capacidad para llevar a cabo ciertos desempeños, compone lo que se llama autoeficacia percibida, la cual se diferencia del locus de control en que además de referirse a las creencias de las personas sobre los resultados de sus acciones, comprende también las creencias acerca de las propias competencias.

\section{La necesidad de control}

El autocontrol puede relacionarse también con el campo motivacional, en cuyo caso toma del nombre de necesidad de control, a la luz de la evidencia de que los individuos comúnmente requieren percibir cierto control sobre su medio ambiente, posiblemente tras experimentar los efectos negativos que su ausencia produce. Constatado eso puede verse cuántos recursos invierte la gente en restaurar el control cuando percibe que aquél está siendo amenazado o recortado. El poder puede ser considerado como una manifestación social de la necesidad de control, dado que los individuos con alta motivación de poder tienden a influenciar sobre decisiones ajenas, a comportarse de manera asertiva, competitiva y agresiva, a buscar de prestigio y a utilizar a los demás en beneficio propio (Peña, 2004).

\section{AUTOCONTROL Y REPERTORIOS DE AFRONTAMIENTO}

A nivel aplicado, se entiende que "El autocontrol se refiere a aquellos procedimientos de terapia cuyo objetivo es enseñar a la persona estrategias para controlar o modificar su propia conducta a través de distintas situaciones, con el propósito de alcanzar metas a largo plazo" (Rehm, 1991: 655). Eso está en consonancia con la enseñanza de estrategias preventivas y de afrontamiento autónomo en el mundo real, de los problemas que aquejan al cliente psicoterapéutico. Las competencias auspiciadas en él a manera de modos de "saber 
hacer" fuera de la sesión de terapia, son la autovigilancia y la relajación, las destrezas de afrontamiento, y cambios específicos en el estilo de vida, con el fin de aprender a disminuir comportamientos excesivos (por ejemplo, adicciones diversas), incrementar comportamientos deficitarios (por ejemplo habilidades sociales y domésticas), o cambiar respuestas inadecuadas (vgr.: no asumir responsabilidades).

Aunque hay diferentes fuentes teóricas que aportan a una perspectiva de autocontrol en psicoterapia, las principales alternativas tecnológicas del análisis conductual que recogen tales procedimientos son las terapias de manejo de la ansiedad y la ira, de asertividad, de condicionamiento encubierto, de reestructuración racional, de solución de problemas, autoinstruccional y de biorretroalimentación, además de otras prácticas incorporadas a ellas, como el manejo contingencial, la comunicación y negociación, la toma de decisiones, etc. Todas ellas actúan con diverso énfasis mediante la modificación de respuestas instrumentales y/o respondientes, cognitivas y afectivas (creencias, pensamientos, actitudes, expectativas, percepciones), además de motoras y fisiológicas.

El entrenamiento en manejo de la ansiedad y la ira propende a reconocer las situaciones, síntomas fisiológicos e interpretaciones que activan dichas emociones impidiendo una recepción normal de mensajes, contrarrestándolas con la aplicación de control autoinstruccional, imaginería, respiración diafragmática y relajación muscular autónoma y entrenamiento en respuestas pro sociales (González, 1992; Suinn, 1990, 1993).

El entrenamiento asertivo comprende métodos como la enseñanza de una adecuada discriminación de situaciones, y de habilidades sociales para aprender a defender los derechos individuales legítimos sin dañar a nadie ni ser agredido (Wolpe, 1958/1980).

El condicionamiento encubierto comprende técnicas de reforzamiento positivo y negativo, castigo, extinción, costo de respuesta y modelado encubiertos, bajo el supuesto de que los principios conductuales son homogéneos tanto a nivel manifiesto como no manifiesto, pudiendo articularse estrategias de uso de tales principios a cargo del propio individuo (Cautela, 1977, 1983).

Las modalidades de reestructuración racional de Ellis y Beck utilizan medios contraargumentativos para que la gente identifique autoafirmaciones incapacitantes y pueda combatirlas autorreforzando respuestas lingüísticas y lógicas incompatibles con el discurso “distorsionado" (véase Yankura y Dryden, 1993, 2000).

La solución de problemas implica procederes de reconocimiento y análisis de los componentes de las situaciones vistas como problemáticas, para perfilar posibles soluciones a ellas mediante decisiones que tengan en cuenta la maximización de beneficios y minimización de costos. (D’ Zurilla, 1986, 1993).

El entrenamiento autoinstruccional incide sobre el autocontrol del "diálogo interno" que utiliza el individuo muchas veces para guiar sus acciones. Para ello analiza funcionalmente las habilidades requeridas para cumplimentar cada acción y programa discursos específicos que faciliten la tarea (Meinchembaum, 1985, 1987).

Por último, la biorretroalimentación se dirige hacia la preparación exhaustiva del individuo para reconocer estados fisiológicos que normalmente pasan inadvertidos, amplificando la 
información de las respuestas mediante aparatos, y aprender a controlar así las propias reacciones de tensión muscular, temperatura, superficie dérmica, actividad cerebral, conductividad de la piel, presión sanguínea y tasa cardíaca (Davis, Robbins y McKay, 1982, 1985).

Los pasos o etapas comunes sobre las que se aplican los procedimientos terapéuticos conductuales antes señalados incluyen el autorregistro, la autoevaluación y el cambio contingencial. La primera etapa permite hacerse consciente de las dimensiones de las conductas problemáticas y otras colaterales en el momento en que suceden; la segunda permite examinar las variables paramétricas registradas (probabilidad, topografía, fuerza y escenario de la conducta) y proponer alternativas de autocontrol secuenciadas en metas, objetivos y análisis de tareas; y la tercera consiste de acciones programadas para autorreforzar, autocastigar o autoextinguir aquellas respuestas consideradas clave en la evaluación de las fortalezas y debilidades implicadas.

\section{COMENTARIO FINAL}

El individuo puede constituirse como un eficaz agente de cambio de su propio comportamiento, como se ha demostrado largamente a través del uso de procedimientos para fomentar la autodirección personal del cliente en el transcurso del episodio psicoterapéutico. Por ello es necesario incentivar la visión del usuario del servicio psicológico en términos de participante activo y responsable, entendiendo que los principios del aprendizaje siguen operando igual en la construcción de repertorios de autocontrol. (Naturalmente que hay individuos cuyo nivel no se sujeta ortodoxamente a este tipo de razonamiento: autistas, perturbados emocionales, esquizofrénicos, retardados y preverbales. Con ellos tendrán que articularse estrategias diferenciales en diversos grados.)

Lo que predomina hoy en día es el tratamiento de la metaconducta, es decir aquella parte del repertorio comportamental que controla a la otra parte. La conducta verbal tiene mucho que ver aquí, por su carácter de repertorio de búsqueda y selección de estimulación discriminativa (conducta guiada por reglas), y por ello tiene un papel fundamental en el autocontrol. En dicho proceso, como dicen López, Rodríguez-Naranjo y Gavino (2006): "El papel del terapeuta es el de ayudar a desarrollar y fortalecer las conductas controladoras" (p. 162), atendiendo y reforzando positivamente su ocurrencia.

\section{REFERENCIAS BIBLIOGRÁFICAS}

1. Ameriks, J., Caplin, A., Leahy, J. \& Tyler, T. (may, 2004). Measuring self-control. National Bureau of Economic Research, Working Paper $N^{\circ} 10514$. Recuperado de: http://www.nber.org/papers/w10514.pdf

2. Ardoy, J. (2007). Control de la conducta inducida por programa por sus consecuencias ambientales. Tesis doctoral, Universidad Nacional de Educación a Distancia, Madrid. Recuperado de: http://e-spacio.uned.es/fez/view.php?pid=bibliuned:20815 
3. Avia, M. D (1995). "El yo privado y el individualismo. Consideraciones históricas y culturales”. En M. D Avia, y M. L. Sanchez (Eds.). Personalidad: aspectos cognitivos y sociales (pp. 107-123). Madrid: Pirámide.

4. Bandura $(1986,1987)$. Pensamiento y acción. Barcelona: Martínez Roca.

5. Campos, L. F. (1973). Diccionario de psicología del aprendizaje. México: ECCSA.

6. Cautela, J. R. (1977/1983). Condicionamiento encubierto: Supuestos básicos y procedimientos. En D. Upper y J. R. Cautela (Eds.). Condicionamiento encubierto (pp. 11-23). Bilbao: Descleé de Brouwer.

7. Davis, M., Robbins, E. \& McKay, M. (198/1985). Técnicas de autocontrol emocional. Barcelona: Martínez Roca.

8. D’Zurilla, Th. J. (1986, 1993). Terapia de resolución de conflictos. Bilbao, Descleé de Brouwer.

9. González, M.D. (1992). Conducta prosocial. Evaluación e intervención. Madrid: Morata.

10. Green, L., \& Estle, S. J. (2003). Preference reversals with food and water reinforcers in rats. Journal of the Experimental Analysis of Behavior, 79, 233-242.

11. Grosch J., \& Neuringer, A. (1981). Self-control in pigeons under the Mischel paradigm. Journal of the Experimental Analysis of Behavior, 35, 3-21.

12. Jackson, K., \& Hackenberg, T. D., (1996). Token reinforcement, choice, and selfcontrol in pigeons. Journal of the Experimental Analysis of Behavior, 66, 29-49.

13. Kanfer, F. H. y Phillips, J. S. (1970/1980). Principios de aprendizaje en la terapia del comportamiento. México: Trillas.

14. Logue, A.W. (1995). Self-Control: Waiting Until Tomorrow For What You Want Today. Englewood Cliffs, NJ: Prentice Hall.

15. López, A. E., Rodríguez-Naranjo, C. y Gavino, A. (2006). Paquetes de técnicas dirigidas a la autorregulación de la conducta y al afrontamiento del estrés. En A. Gavino (Coord.). Guía de técnicas de terapia de conducta (pp. 159-201). Madrid: Pirámide.

16. Meinchembaum, D. H. (1985/1987). Manual de inoculación de estrés. Barcelona: Martínez Roca.

17. Montgomery, W. (1997). “Terapia conductual y modelo de afrontamiento: En busca del cliente autónomo". Revista de Psicología de la Universidad Nacional Mayor de San Marcos, I(1), 65-72.

18. Montgomery, W. (2002). La comunicación en el episodio psicoterapéutico: Algunas formas referenciales. En Comportamiento lingüístico: Temas selectos (pp. 133-150). Lima: Asociación Peruana de Psicología Interconductual.

19. Nevin, J. A. (2008). Control, prediction, order, and the joys of research. Journal of Experimental Analisis of Behavior, 89(1), 119-123. 
20. Peña. D. (2004). Necesidad de control: Análisis conceptual y propuesta experimental. Revista Profesional Española de Terapia Cognitivo-Conductual, 2, 70- 91.

21. Rasmussen, E. B. y Newland, M. C. (2008). Asymmetry of Reinforcement and Punishment in Human Choice. Journal of Experimental Análisis of Behavior; 89(2): 157-167.

22. Rehm, L. (1991). "Métodos de autocontrol”. En V. Caballo (Ed.). Manual de modificación y terapia del comportamiento (pp. 655-683). Madrid: Pirámide.

23. Skinner, B. F. (1953/1971). Ciencia y conducta humana. Barcelona: Fontanella.

24. Skinner, B. F. (1979(1980). Autobiografía, Vol. 3. Cómo se forma un conductista. Barcelona: Fontanella.

25. Staats, A. W. (1975/1979). Conductismo social. México: El manual moderno.

26. Suinn, R. M. (1990/1993). Entrenamiento en manejo de ansiedad. Bilbao: Descleé de Brouwer.

27. Thoresen, C. E. y Mahoney, M. J. (1974/1980). Conducta y autocontrol. Madrid: Morata.

28. Visdómine, J.C. y Luciaño, C. (2006). Locus de control y autorregulación conductual. Revisiones conceptual y experimental. International Journal of Clinical and Health Psychology, 6(3), 729-751.

29. Wolpe, J. (1958, 1980). Terapia por inhibición recíproca. Bilbao: Descleé de Brouwer.

30. Yankura, J. y Dryden, W. (Comps.) (1993/2000). Terapia conductual racional emotiva: Casos ilustrativos. Bilbao: Descleé de Brouwer. 